\title{
SAFA:A new measure to evaluate psychiatric symptoms detected in a sample of children and adolescents affected by eating disorders. Correlations with risk factors
}

\author{
Emilo Franzoni' \\ Morena Monti' \\ Alessandro Pellicciari' \\ Carlo Muratore \\ Alberto Verrotti ${ }^{3}$ \\ Caterina Garone \\ Ilaria Cecconi' \\ Luisa lero' \\ Stefano Gualandi ${ }^{2}$ \\ Francesca Savarino' \\ Paola Gualandi' \\ 'Child Neuropsychiatry Unit, \\ Clinical Pediatrics; ${ }^{2}$ Protection \\ and Enhancement Department, \\ University of Bologna, Italy; ${ }^{3}$ Clinic \\ of Paediatrics, University of Chieti, \\ Chieti, Italy
}

Correspondence: Emilio Franzoni Via Massarenti II - 40I 38 Bologna, Italy $\mathrm{Tel}+3905$ I 6363 653, Ext 346744 $\mathrm{Fax}+3905$ I 304839

Email emilio.franzoni@unibo.it

\begin{abstract}
In order to evaluate the psychiatric symptoms associated with a diagnosis of eating disorders (ED) we have administered a new psychometric instrument: the Self Administrated Psychiatric Scales for Children and Adolescents (SAFA) test. SAFA was administered to a cohort of 97 patients, aged from 8.8 to 18, with an ED diagnosis. Age, body mass index (BMI) and BMI standard deviation score were analyzed. Furthermore, while looking for linkable risk factors, we evaluated other data that took an influence over the SAFA profile, like parental separation and family components' number. Compared to the range of statistical normality (based on Italian population), patients with bulimia nervosa or binge-eating disorder showed higher and pathologic values in specific subscales. When analyzing sex, males showed more pathologic values in most anxiety-related, obsessiveness-compulsiveness-related and insecurity subscales. A correlation among age, BMI and specific subscales (low self esteem, psychological aspects) emerged in participants with anorexia nervosa. In order to plan more appropriate diagnostic and therapeutic approaches in children or adolescents suffering from ED, the SAFA test can be an important instrument to evaluate psychiatric symptoms. Therefore, we propose to include this useful, simple self-administered test as a new screening tool for ED diagnosis.
\end{abstract}

Keywords: psychiatric comorbidity, anorexia nervosa, bulimia nervosa, binge-eating disorder, SAFA test

\section{Introduction}

Eating disorders (ED) are an important cause of physical and psychosocial morbidity in adolescents and young adults. ${ }^{1}$

The Diagnostic and Statistical Manual of Mental Disorders (DSM-IV) classified ED in anorexia nervosa (AN), bulimia nervosa (BN) and eating disorders not otherwise specified (EDNOS). AN and BN are characterized by aberrant patterns of eating behavior and weight regulation and by disturbances in attitudes towards weight and perception of body shape., ${ }^{2,3}$

The refusal of a body weight higher than the minimal appropriate for age is a typical feature of $\mathrm{AN}$, while $\mathrm{BN}$ is characterized by recurrent binge episodes followed by inappropriate purging behaviors, such as self-induced vomiting, laxatives, diuretics, and excessive physical activity. ${ }^{2}$

EDNOS are ED that do not apply to the diagnostic criteria for AN or BN. ${ }^{1}$ Bingeeating disorder (BED) is included as a subcategory of EDNOS; its defining feature is an introduction of a large amount of food in a relatively short period of time with feelings of loss of control. Other associated features include eating rapidly, eating until uncomfortably full, eating alone because of embarrassment, eating large amounts of 
food when not physically hungry, and feeling disgusted or guilty after binge eating. ${ }^{2,4}$

A prevalence average rate for $\mathrm{AN}$ of $0.3 \%$ is reported in young females. The prevalence rate for $\mathrm{BN}$ is $1 \%$ and $0.1 \%$ for young women and young men respectively while the estimated prevalence of BED is at least $1 \% .^{5}$ Advances in pathogenesis of ED have proposed a multidimensional mechanism that recognizes:

1) biological, psychological, and sociocultural risk factors;

2) causing factors such as a stressful event;

3) elements of positive reinforcement;

4) secondary effects of food restriction.

The management of ED provides psychological, nutritional, and pharmacological approaches. ${ }^{1,6}$

Psychiatric comorbidity with Axis I (depression, obsessivecompulsive disorder, substance abuse) and Axis II (personality disorders) often complicate case conceptualization and treatment planning. ${ }^{7}$

These patterns can be revealed by clinical interviewbased methods and psychometric evaluations. Although interview-based methods have advantages in terms of greater diagnostic specificity, they can lack convenience in a research setting where psychometric evaluations can provide quantitative information about the severity of the problem.

The aim of this study is to evaluate psychiatric disorder symptoms and their relationships with the subtypes of ED. In order to achieve this purpose, we used an Italian instrument: Self Administrated Psychiatric Scales for Children and Adolescents (SAFA) test. ${ }^{8}$ SAFA has not been widely translated or validated in other languages, therefore we aim to reach a wider range of researchers and/or clinicians for whom this instrument may be pertinent.

Moreover, in this study we have identified as risk factors some social aspects that are increasing in relevance in the Italian families (parental separation, presence of only children). We looked for correlations with specific comorbidity profiles found with the SAFA test.

\section{Materials and methods}

\section{Participants}

We consecutively enrolled 97 patients, 87 females and 10 males, with an average age of 15.1 (SD: 2.3, range 8.8-18) and diagnosis of ED, admitted to our ED Center. Sixty-five of 97 (67\%) were diagnosed as AN, 18/97 (18.6\%) as BN, and 14/97 (14.4\%) as BED.

Our patients were given general information about the aims of our study and we asked for parental consent before starting the administration. None of the patients were given payment and none were forced to participate in the scale.

\section{Procedures}

\section{Inclusion criteria}

Diagnosis of AN, BN, or BED according to DSM-IV criteria.

\section{Exclusion criteria}

Age $<8$ years and age $>18$ years with difficulty concentrating due to very severe malnutrition, and patients already treated with pharmacological or psychotherapeutic approaches at the time of the administration.

The data was collected between March 2005 and June 2008 .

The diagnosis of ED was completed by clinical assessment, evaluation of body mass index (BMI), physical and nutritional examination, and psychological interview at the patient's admittance to our Neuropsychiatry Unit.

The SAFA test was administered to every patient individually in a quiet, comfortable and illuminated setting.

\section{SAFA}

SAFA is an Italian psychometric test processed by C. Cianchetti and G. Sannio Fascello, and was published in 2001. It is intended for children and adolescents aged 8 to $18 .^{8}$ SAFA has been validated by Special Organizations (S.O.) in Florence (2001), having previously undergone standardization on a sample of 895 healthy participants (442 aged 8-10, 265 aged 11-13, and 188 aged 14-18) and having been comparatively administered to 125 patients with different psychiatric pathologies assessed by the Neuropsychiatry Clinic of Cagliari (41 aged 8-10, 42 aged 11-13, and 42 aged 14-18).

SAFA is offered as a unitary instrument that allows a preliminary but sufficiently broad assessment of psychiatric conditions by means of different scales that are organized according to homogeneous criteria. This battery allows examiners to explore a wide series of symptoms and psychiatric conditions in a short period of time. On the basis of the scores obtained, it is possible to build a general profile and/or individual profiles within the single scales, according to the main groups of the explored symptoms. Successive administrations can provide useful information to assess the effectiveness of therapeutic intervention.

The test is structured to adapt itself to the comprehension level and assessment methods of all age groups. Therefore, each scale consists of a version for subjects aged 8-10 (identified by the letter " $\mathrm{e}$ ") and by a single version for subjects aged $11-18$ years (identified by " $\mathrm{m} / \mathrm{s}$ "); the only exception 
is the anxiety scale which presents three different versions: 8-10 years ("e"), 11-13 years ("m"), and 14-18 years ("s").

The administration can be both individual and collective (for example, screening in schools), lasts between 30 and 60 minutes, and includes a total of six scales (each with subscales) that can also be used separately.

\section{Scale for evaluation of anxiety (SAFA A)}

- Generalized anxiety (A1), meaning tension/uneasiness (4 items in SAFA e and 6 in SAFA m/s) and apprehensiveness/ preoccupations about the future ( 6 items in each version)

- Social anxiety (A2) (10 items in SAFA e and 12 in SAFA $\mathrm{m} / \mathrm{s}$ ), which investigates the characteristics of the avoidant disorder [DSM III R]

- Separation anxiety (A3), which investigates separation in the literal sense ( 5 items in SAFA e and 6 in $\mathrm{m}$ and $\mathrm{s}$ ), apprehension about loss ( 3 in SAFA e and 4 in $\mathrm{m} / \mathrm{s}$ ) and abandonment (2 items)

- School-related anxiety (A4) with 10 items in SAFA e and 12 in SAFA m/s

\section{Scale for evaluation of depression (SAFA D)}

SAFA D follows the DSM IV criteria for diagnosis of major depressive episode and dysthymic disorder. Therefore it explores depressed mood (D1), anhedony and disinterest (D2), touchy mood (D3), sense of inadequacy and low self esteem (D4), insecurity (D5), guilt (D6), and hopelessness (D7). No items investigate somatic symptoms such as weigh variations, sleep difficulties, asthenia, difficulties of concentration (which are objective data or items included in SAFA S), while evaluation of psychomotor slackening is made by the clinic according to DSM-IV. Finally the authors chose not to investigate a critical matter like suicidal ideation with a psychometric instrument.

\section{Scale for evaluation of obsessive- compulsive symptoms (SAFA O)}

SAFA O considers as subscales, obsessive thoughts $(\mathrm{O} 1)$, compulsions and rituals $(\mathrm{O} 2)$, rupophobia and contamination (O3), order and control (O4), and doubt and indecision (O5). It includes 38 items altogether in the " $\mathrm{m} / \mathrm{s}$ " version and 20 in the "e" version.

\section{Scale for evaluation of psychogenic eating disorders (SAFA P)}

SAFA P " $\mathrm{e}$ " is constituted by 20 items and SAFA P " $\mathrm{m} / \mathrm{s}$ " by 30 . Items are related by bulimic behavior (P1), anorexic behavior (P2), acceptance and evaluation of one's own body (P3), and psychological aspects (P4: fear of maturity, perfectionism, and inadequacy; SAFA "e" is more focused on these themes due to the necessity of exploring psychological risk factors when a clear eating disorder is quite rare at $8-10$ years of age). A further subscale considers the sum of anorexic behavior and acceptance/evaluation of own body (P2P3).

\section{Scale for evaluation of somatic symptoms and hypochondria (SAFA S)}

With 20 items in the "e" version and 25 in "m/s", SAFA S evaluates symptoms related with: cardiac, gastrointestinal and respiratory systems, asthenia, sleep, general cenesthesis, and memory/concentration.

\section{Scale for evaluation of phobias (SAFA F)}

Regarding specific phobias, SAFA F identifies and values 24 different types of fears. The authors determined which phobias were to be chosen according to their statistical relevance in a sample of 180 subjects. However, an "other" possibility is provided.

Each item has three possible choices: "true, false, and partly true." SAFA $\mathrm{S}$ is the only scale that offers "often, sometimes, and never" as possible answers.

Each item can be valued from 0 to 2 according to specific correction schedules and the sum of points achieved in each scale and subscale can be converted into $\mathrm{T}$ scores using reference tables for age and sex $(T=50+10 Z)$. $T$ scores define different ranges:

- $\quad$ 30: the domain investigated is negated by the subject

- 30-39: the subject tends to negate the symptoms

- 40-59: statistical normality

- 60-69: symptoms are basically pathologic

- >69: symptoms are pathologic

In order to value the accuracy and the trustworthiness of the answers, SAFA has two instruments:

- A simulation scale is provided and included in the SAFA D scale as a subscale. One or more positive answers in these items (such as "I've never lied" or "I've always studied every school lesson") suggest doubts about the whole test validity, since the subject has not shown sincerity, or engagement, or an adequate comprehension of the items.

- A couple of items are repeated in three different scales (SAFA P, A, and O): these items are related with the SAFA O scale, but work as a control in the others. Therefore accuracy and trustworthiness of the battery can be examined, even if SAFA D is not administered. 
With regard to the psychometric properties of the battery, internal consistence and stability are respected, as SAFA shows a Cronbach's $\alpha$ coefficient and a split half $>0.80$, while indices of each scale at the one week test-retest procedure showed a $\mathrm{p}<0.01$. Convergent validity was respected comparing SAFA A with Anxiety Scale Questionnaire (ASQ) ${ }^{9}$ and with "Questionario Scala Ansia per l'Età Evolutiva," an Italian instrument addressed to children and adolescents aged 8-16. ${ }^{10}$ SAFA D was compared with Children's Depression Inventory (CDI), ${ }^{11}$ and with the Clinical Depression Questionnaire (CDQ). ${ }^{12}$ Analysis of Pearson's $r$ coefficient showed a statistically significant correlation $(p<0.001)$. Discriminatory validity was obtained through a deep selection of the items (that were 180 in each scale), choosing, in the final version of SAFA, only those that resulted higher in pathological subjects than in control group $(\mathrm{p}<0.001)$. Finally, content validity was verified through the correlation between single items and total score.

Since this measure has not been widely translated and validated, only a few previous researches including SAFA have been published in English language-reviewed journals. An interesting research used SAFA to demonstrate higher levels of anxiety and depression in obese adolescents carrying a peculiar mutation in the CART gene when compared with patients without the mutation, who were in the same age group and had the same degree of obesity. ${ }^{13}$

\section{Statistical analysis}

We used the computer program Statistical Package for Social Science 14.0 (SPSS Inc., Chicago, IL) integrated with SPSS exact test module for statistical analysis. The data distribution was analyzed with skewness and kurtosis coefficients and Kolmogorov-Smirnov test.

Normally distributed data are expressed as means $\pm \mathrm{SD}$, and nonnormally distributed data as median and interquartile range (IQR, which is the distance between 25 th and 75 th percentile; it includes the middle $50 \%$ of the observation). When possible, a normal distribution was obtained after logarithmic transformation of nonnormally distributed data.

In normally distributed data the statistical significance was processed using Student's t test, one-way analysis of variance for multiple comparisons (Bonferroni test), Pearson's correlation index and multiple regression analysis. For abnormally distributed data the Mann-Whitney and Kruscal-Wallis tests, the Spearman rank correlation, Chi-square and Fisher's exact tests were used. While working on small samples the SPSS exact test was used. A p-value $<0.05$ (two tailed) was considered as a significant value.
BMI and SAFA subscales were standardized with a reference population, homogeneous for age and sex. BMI was transformed in correspondent standard deviation score point $Z$ while scales and subscales of SAFA were converted into correspondent $\mathrm{T}$ scores.

\section{Results \\ Demographic analysis}

The AN group is constituted by 58 females and 7 males, with an average age of $14.9 \pm 2.4$ years (range $8.8-18$ ), an average BMI of 15.3 and an average BMI standard deviation score (BMIsds) of $-2.75 \pm 1.33$ [range $-6--0.58$ ]. The group of patients with $\mathrm{BN}$ is composed of 18 females, with an average age of $16.5 \pm 1.30$ years (range 13.4-18.6) and an average BMIsds of $-0.32 \pm 1.42$ (range $-2.65-2.37$ ), while the group with BED was of 11 females and 3 males, aged $14.4 \pm 2.2$ in mean and with an average BMIsds of $2.60 \pm 0.92$ (range 0.56-4.08).

By analyzing average ages with Bonferroni's test, we find that bulimic patients are older than the other groups ( $p<0.05)$, a datum supported by literature: Klein and Walsh described as typical the onset of BN during the late adolescence. ${ }^{14}$ This may explain the disproportion between anorexic and bulimic patients, as our ED center is focused on children and adolescents. The second cause of disproportion can be the selection made to admit to the ward, choosing patients with very low weights.

\section{Psychiatric symptoms}

By analyzing the scales and subscales of SAFA, we noticed characteristic pathologic scores that deserve to be mentioned. By using Bonferroni's test and comparing all scales and subscales, we report a statistically significant difference $(\mathrm{p}<0.0001)$ involving subscale P1 (bulimic behavior), where we observe high values in $\mathrm{BN}$ and $\mathrm{BED}$ groups if compared with the $\mathrm{AN}$ group. In particular, BNs show median values of 65 , BEDs of 61, and ANs of 42. A statistically significant difference ( $p$ $<0.001$ ) is found by analyzing subscale P3 (acceptance and evaluation of own body): we observe elevated values in the BED group (Median: 70), while BNs have median values of 57 and AN of 47 (Table 1).

The percentage of subjects showing pathologic values in the $\mathrm{P} 1$ subscale is significantly higher $(\mathrm{p}<0.0001)$ in the BED group (10/14 with $\mathrm{T} \geq 60,71 \%$ ), than in the $\mathrm{BN}$ group $(10 / 18,56 \%)$ and in the AN one $(3 / 62$, which shows a very low percentage of $5 \%$ ).

The same occurs with P3, where the percentage of participants showing pathologic values results significantly higher 
Table I Psychiatric symptoms and pathologic values

\begin{tabular}{|c|c|c|c|}
\hline & AN (n 65) & BN (n I6) & BED (n I4) \\
\hline A2 (Social anxiety) & $60(50-7 I)$ & $54(44-73)$ & $57(43-73)$ \\
\hline D2 (Anhedony and indifference) & $56(46-7 I)$ & $62(48-76)$ & $49(46-58)$ \\
\hline D4 (Sense of inadequacy, low self esteem) & $58(49-73)$ & $62(53-72)$ & $56(47-67)$ \\
\hline PI (Bulimic behavior)* & $42(42-50)$ & $65(44-73)$ & $61(52-72)$ \\
\hline $\begin{array}{l}\text { P3 (Acceptance and evaluation of one's } \\
\text { own body)* }\end{array}$ & $47(42-6 I)$ & $57(35-70)$ & $70(65-7 I)$ \\
\hline $\begin{array}{l}\text { P2P3 (Anorexic behavior + acceptance } \\
\text { and evaluation of one's own body) }\end{array}$ & $55(46-68)$ & $61(50-70)$ & $61(55-69)$ \\
\hline
\end{tabular}

Notes: The table shows median (IQR) values of pathologic subscales in the three groups; ${ }^{*} \mathrm{p}<0.000 \mathrm{I}$.

Abbreviations: AN, anorexia nervosa; $\mathrm{BED}$, binge-eating disorder; $\mathrm{BN}$, bulimia nervosa.

$(\mathrm{p}<0.0001)$ in the BED group $(12 / 14,86 \%)$ in comparison with BN $(8 / 18,44 \%)$ and AN $(18 / 65,28 \%)$.

Furthermore, we focus on subscales with pathologic or basically pathologic results: we observe homogeneous profiles among the three groups, involving subscales A2 (social anxiety), D (depression), D2 (anhedony), D4 (low self esteem), and P2P3. These values do not reach a statistically significant difference, but we can notice how the $\mathrm{T}$ scores tend to be greater than 60 (Table 1).

\section{Risk factors}

We notice that the percentage of parental separation in the three groups is importantly higher than the average of the Northern Italy population, which is esteemed in 10.2 (6.2 separations and 4 divorces) per 1000 married couples. ${ }^{15} \mathrm{We}$ observe a percentage of separated parents of $36 \%$ in the BED group, $26 \%$ in the BNs, and $14 \%$ in the ANs $(21 \%$ considering DCAs altogether).

Therefore, we looked for characteristic profiles involving this datum at the SAFA administration. Considering the ANs group, Student's t test and Mann-Whitney test point out that elevated scores in the social anxiety (A2) and school-related anxiety (A4) subscales are connected with parental separation in a statistically significant manner $(\mathrm{p}<0.05)$.

Considering the other subscales with pathological meaning, they do not show statistically significant differences amongst the two groups. However we can point out basically higher scores in the separated group, with regards to several areas of depression and determined psychological aspects, like fear of maturity, inadequacy, and perfectionism (for example, depression scale is 71 in separated group and 58 in not separated group; Table 2).

BNs do not show significant findings, but BEDs show statistically significant differences in the P scale (psychogenic eating disorders), which are higher in the group with separated parents (P medians: 69 vs 54, $\mathrm{p}<0.05$ ).

By analyzing with Mann-Whitney test the number of family members, we split the cohort into two groups: one with three or less members and one with more.

Anorexic patients belonging to larger families show significantly higher values in the sense of guilt subscales (D6), showing a median value of 62 when only children presented 50. On the other hand, patients with family number of three or less show higher values and pathologic rates in the subscales A2 (social anxiety) and D1 (depressed mood, 62 vs 57). We consider this latter datum interesting even if it does not reach a statistical significance.

Simulation scores are higher in participants with restricted families, both in the total cohort of patients (37\% vs $15 \%)$ and in the ANs $(50 \%$ vs $20.5 \%)(\mathrm{p}<0.05)$.

More data can be achieved by analyzing the sex of participants with the Mann-Whitney test and Student's t test: anorexic males seem to have a more affected profile. In particular, males show significantly higher scores in the anxiety-related scales and subscales (A, A3, A4, p $<0.005$, and A1, p $<0.0001$ ). We also find statistically significant differences in the scales investigating obsessiveness and compulsiveness $(\mathrm{O}, \mathrm{O} 1$, and $\mathrm{O} 2)$ and insecurity (D5) $(\mathrm{p}<0.05)$. Even if the sample is quite scant, these data seem to bring useful information (for example, generalized anxiety 74 in males vs 52 in females, Table 3).

Finally, age and low self esteem (D4) seem to act as an aggravating factor in anorexia. We notice a negative correlation between these factors and BMI ( $p<0.005$ ). In fact, the dependent variable of BMI was analyzed with several explanatory variables, namely age, D (depression), D4 (low self esteem), D7 (hopelessness) and P4 (psychological aspects). Multiple regression analysis reveals a significant inverse correlation between BMIsds and D4 $\left(\mathrm{r}^{2}=0.14\right)$; 
Table 2 Pathologic profiles involving parental separation; anorexia and binge-eating disorder (BED) (last value)

\begin{tabular}{lll}
\hline Anorexia & Separated (n 9) & Nonseparated (n 56) \\
\hline A2 (Social anxiety) & $73^{*}(55-75)$ & $57(49-70)$ \\
A4 (School-Related anxiety) & $53^{*}(50-57)$ & $46(39-53)$ \\
D (Depression) & $71(51-75)$ & $58(47-70)$ \\
DI (Depressed mood) & $62(49-73)$ & $59(47-68)$ \\
D2 (Anhedony and indifference) & $62(48-76)$ & $52(46-69)$ \\
D4 (Sense of inadequacy and low self esteem) & $66(47-74)$ & $57(49-69)$ \\
D7 (Hopelessness) & $67(45-74)$ & $51(42-65)$ \\
P2 (Anorexic behavior) & $65(46-70)$ & $58(45-68)$ \\
P4 (Psychological aspects) & $60(43-69)$ & $57(45-65)$ \\
P2P3 (Anorexic behavior + acceptance and & $60(46-72)$ & $54(45-67)$ \\
evaluation of one's own body) & & Nonseparated (n.9) \\
BED & Separated (n.5) & $54(51-61)$ \\
P (Psychogenic eating disorders)* & $69(70-52)$ & \\
\hline
\end{tabular}

Notes: Anorexic and BED patients with parental separation show higher values in specific subscales; ${ }^{*} \mathrm{p}<0.05$ vs nonseparated group.

age is the second variable that shows a significant negative correlation with BMIsds $\left(r^{2}=0.07\right)$. Multiple regression analysis explains the correlation among BMIsds and D, D7 and $\mathrm{P} 4$ as a covariation of D4 (Table 4).

\section{Discussion}

The SAFA test does not make a diagnosis of ED or psychiatric comorbidity. Nevertheless, it gives a valuable method for the patients' evaluation, clarifying diagnostic doubts, and suggesting referring points for further investigations.

Aspects of the psychological conditions (as anxiety or depressive mood) can be easily and practically analyzed. This first evaluation can be very useful in order to plan diagnostic and therapeutic approaches in children and adolescents with ED.

Even if our study is not exempt from limits (such as the small sample and the disproportion between AN group and the other two), interesting data can be drawn to attention.
Since previous studies reported the presence of psychiatric comorbidity as an aggravating factor in case conceptualization and treatment planning of $\mathrm{EDs}^{7}$ and since Speranza and colleagues ${ }^{16}$ found a statistically significant incidence of depression (evaluated by the Beck Depression Inventory) in EDs if compared with controls, we used SAFA test to investigate psychological traits in our cohort.

We report homogeneous characteristics among patients with ED: the elevation of anxious and depressive traits, with values belonging to the "basically pathologic" range.

Psychiatric comorbidity appears more serious with regard to the male population, where we find a statistically significant difference when males are compared with females in anxiety, obsessiveness, and insecurity areas. Therefore SAFA suggests a more affected profile in anorexic males, a finding that can be investigated with further studies.

Table 3 Influence of sex over anorexia

\begin{tabular}{llll}
\hline & Males (n 7) & Females (n 58) & P \\
\hline A (Scale for evaluation of anxiety) & $66(60-74)$ & 5 I (44-60) & $<0.005$ \\
AI (Generalized anxiety) & $74(65-74)$ & $52(44-6 I)$ & $<0.000$ I \\
A3 (Separation anxiety) & $65(55-73)$ & $48(39-58)$ & $<0.005$ \\
A4 (School-related anxiety) & $62(50-7 I)$ & $46(40-53)$ & $<0.005$ \\
D5 (Insecurity) & $64(6 \mathrm{I}-68)$ & $53(46-62)$ & $<0.05$ \\
O (Scale for evaluation of & $64(50-67)$ & $48(42-56)$ & $<0.05$ \\
obsessive-compulsive symptoms) & & & $<0.05$ \\
OI (Obsessive thoughts) & $60(50-68)$ & $48(4 I-59)$ & $<0.05$ \\
O2 (Rituals and compulsions) & $63(49-68)$ & $47(4 I-58)$ & \\
\hline
\end{tabular}


Table 4 BMI standard deviation score: Correlations with age and specific subscales

\begin{tabular}{ll}
\hline Age & $\mathbf{r}=-\mathbf{0 . 3 8}$ \\
\hline D (Depression) & $\mathbf{p}<\mathbf{0 . 0 0 5}$ \\
\hline D4 (Sense of inadequacy and low self esteem) & $r=-0.27$ \\
& $\mathrm{P}<0.05$ \\
D6 (Guilt) & $\mathrm{r}=-0.37$ \\
& $\mathrm{P}<0.005$ \\
D7 (Hopelessness) & $\mathrm{r}=-0.25$ \\
& $\mathrm{P}<0.05$ \\
P4 (Psychological aspects) & $\mathrm{r}=-0.30$ \\
& $\mathrm{P}<0.05$ \\
\hline
\end{tabular}

Notes: Negative correlations involving BMI with age and specific subscales. As the patient grows affected by the ED, a worse profile and a more negative BMI are noticed.

Abbreviations: BMI, body mass index; ED, eating disorder.

The acceptance and evaluation of one's own body (P3) result pathologically involved in each ED; however SAFA underlines a prevalence of this psychological trait in BED and $\mathrm{BN}$.

Our findings are supported by two previous studies, one demonstrating a negative self-evaluation of patients with BED if compared with healthy participants, and the other showing high scores of BEDs in the EDI 2 body dissatisfaction subscale. ${ }^{17,18}$

Furthermore, we used SAFA to look for risk factors: a correlation among pathologic values in specific subscales and BMI, familiar nucleus and parental separation was sought.

With regard to parental separation, SAFA detects that children and adolescents with ED are likely to show worse psychological functioning (mostly in the anxiety-related area) if the family nucleus is broken, a datum, however, confirmed by literature; Gual and colleagues ${ }^{19}$ underlined the importance of family assessment in ED, since they found that children experiment feelings like low self esteem (predictors of ED) after parental separation; Tripp and Cockett $^{20}$ described higher psychiatric risks caused by peculiar family backgrounds or parental separation and divorces, with low self evaluation, school and social difficulties as early effects.

Moreover, according to SAFA results, the number of family components seems to be of some effect on the patient's functioning, with increased sense of guilt in numerous families and more pathologic symptoms regarding social anxiety and depressed mood when we are facing only children (the latter datum should be only taken as descriptive, since no statistical significance was noticed).

Therefore, once again SAFA reveals the important role of family in the clinical course of ED and in treatment and prognosis consequently.

The last findings show that individuals with lower BMI are older and have a more severe psychopathology: belated observation or diagnoses may involve more structured anorexic/ bulimic features, with important depressive backgrounds. This is information that, if confirmed, would underline the importance of an early diagnosis and treatment plan.

In conclusion, the advantage of SAFA is in "taking a photo" of the whole person as it appears at the time of the administration, without necessity of previous long and expensive training courses, in a small amount of time and in a practical way, and to have an instrument that - we think - may be recommended in the diagnostic flowchart of patients affected by ED.

\section{Disclosure}

The authors report no conflicts of interest in this work.

\section{References}

1. Fairburn CG, Harrison PJ. Eating disorders. Lancet. 2003;361(9355): 407-416.

2. American Psychiatric Association. Diagnostic and Statistical Manual of Mental Disorders (DSMIV). Washington, DC: American Psychiatric Association; 1994

3. Kaye WH, Klump KL, Frank GK, Strober M. Anorexia and bulimia nervosa. Annu Rev Med. 2000;51:299-313.

4. Wheeler K, Greiner P, Boulton M. Exploring alexithymia, depression and binge eating in self-reported eating disorders in women. Perspect Psychiatr Care. 2005;41(3):114-123.

5. Hoek HW, van Hoeken D. Review of the prevalence and incidence of eating disorders. Int J Eat Disord. 2003;34(4):383-396.

6. Zhu AJ, Walsh BT. Pharmacologic treatment of eating disorders. Can J Psychiatry. 2002;47(3):227-234.

7. O'Brien KM, Vincent NK. Psychiatric comorbidity in anorexia and bulimia nervosa: nature, prevalence and causal relationships. Clin Psychol Rev. 2003;23(1):57-74.

8. Cianchetti C, Sannio Fascello G. Scale Psichiatriche di Autosomministrazione per Fanciulli e Adolescenti (SAFA). Organizzazioni Speciali. 2001.

9. Cattel RB, Scheier IH. The IPAT Anxiety Scale Questionnaire. Instit for Personal \& Abil Test, Champagn III, 1963.

10. Busnelli C, Dall'Aglio P, Farina P. Questionario Scala d'Ansia per l'età evolutiva. Finenze; Organizzazioni Speciali, 1974.

11. Kovacs M. Rating Scales to assess depression in school aged children. Acta paedapsychiatrica. 1981;46:305-315.

12. Krug SE, Laughin JE. Clinical Depression Questionnaire. Instit for Personal \& Abil Test, Champagn III, 1976.

13. Miraglia Del Giudice E, Santoro N, Fiumani P, Dominguez G, Kuhar MJ, Perrone L. Adolescents carrying a missense mutation in the CART gene exhibit increased anxiety and depression. Depress Anxiety. 2006;23(2):90-92.

14. Klein DA, Walsh BT. Eating disorders: clinical features and pathophysiology. Physiol Behav. 2004;81:359-374. 
15. ISTAT (Italian National Institute of Statistics). 2005. Cited on January 10, 2009. Available from http://www.istat.it/.

16. Speranza M, Corcos M, Loas G, et al. Depressive personality dimensions and alexithymia in eating disorders. Psychiatry Res. 2005;135(2): 153-163.

17. Marcus MD, Kalarchian MA. Binge eating in children and adolescents. Int J Eat Disord. 2003;34(Suppl):S47-S57.

18. Ramacciotti CE, Coli E, Passaglia C, Lacorte M, Pea E, Dell'Osso L. Binge eating disorder: prevalence and psychopathological features in a clinical sample of obese people in Italy. Psychiatry Res. 2000;94(2): 131-138.
19. Gual P, Pérez-Gaspar M, Martínez-González MA, Lahortiga F, de Irala-Estévez J, Cervera-Enguix S. Self-esteem, personality, and eating disorders: baseline assessment of a prospective population-based cohort. Int J Eat Disord. 2002;31(3):261-273.

20. Tripp JH, Cockett M. Parents, parenting, and family breakdown. Arch Dis Child. 1998;78(2):104-108. 